Classification

Physics Abstracts

$47.15-68.45 \mathrm{Gd}-68.10 \mathrm{Gw}$

\title{
Capillary rise of a wetting fluid in a semi-circular groove
}

\author{
Elie Raphaël \\ Laboratoire de Physique de la Matière Condensée, (associated with the C.N.R.S. U.A 792), \\ Collège de France, 75231 Paris Cedex 05, France
}

(Reçu le 9 juin 1988, révisé le 7 septembre 1988, accepté le 17 octobre 1988)

\begin{abstract}
Résumé. - L'étalement d'un liquide sur une surface solide est modifié si celle-ci présente des cannelures. Nous étudions le profil d'un liquide mouillant qui monte à partir d'un réservoir le long d'un mur vertical comportant une cannelure semi-circulaire. Le profil d'équilibre ainsi que la dynamique d'invasion du liquide dans la cannelure sont calculés.
\end{abstract}

\begin{abstract}
The presence of grooves on solid surfaces modifies the spreading of liquids. We study the profile of a wetting fluid climbing from a reservoir onto a vertical wall with a semi-circular groove. The equilibrium profile and the dynamics of penetration of the liquid inside the groove are calculated.
\end{abstract}

\section{The model.}

The penetration of a fluid into a capillary structure is of considerable importance in a wide range of technological applications (e.g., imhibition in porous media). Since the original studies of Lucas [1] and Washburn [2], a considerable amount of experimental and theoretical work has been devoted to study the flow in capillary tubes [3]. Others geometries have also been considered. In particular, wetting in a corner [4] and the flow along a dihedral [5] have been studied in detail.

Recent experiments [6] seem to indicate that the dynamics of the fluid ascension along a vertical fiber is drastically affected by the presence of grooves on the surface of the fiber. As a first step towards the understanding of the experimental system, we consider the following model problem : a wetting fluid in contact with a vertical wall with a semi-circular vertical groove (Fig. 1). Long-range forces (Van der Waals, electrostatic), though important in many cases, will be neglected throughout this paper.

In the absence of the groove, the liquid forms a macroscopic meniscus of height $\sqrt{2} \kappa^{-1}$ all along the vertical wall $\left(\kappa^{-1}\right.$ is the capillary length $: \kappa^{-1}=\sqrt{\gamma / \rho g}$ where $\gamma$ and $\rho$ are, respectively, the liquid surface tension and density). Since the edges of the groove are sharp, it is reasonable to assume that outside the groove this meniscus remains unperturbed. Inside the groove of radius $R$, the liquid rise is due to surface tension effects. At height $z$ (above the macroscopic meniscus), the liquid forms a sickle-shape lunula characterized by its free surface radius of curvature $r$, thickness $e$, and area $\Sigma$ (Fig. 2). Note that the liquid is 


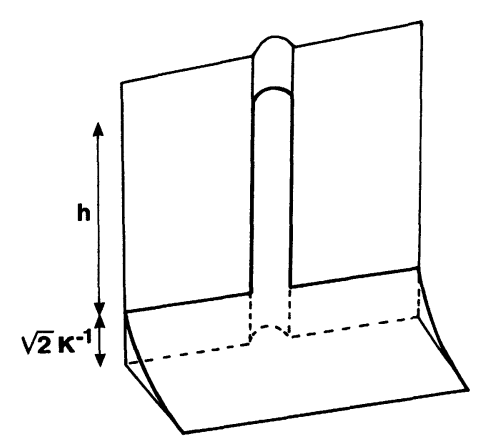

Fig. 1. - Capillary rise of a liquid into a vertical semi-circular groove.

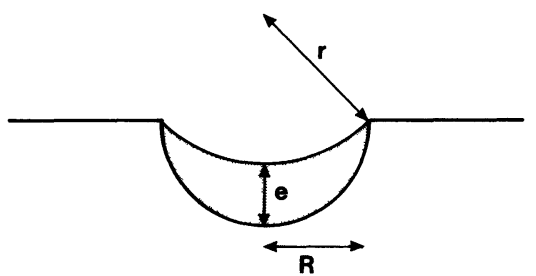

Fig. 2. - Horizontal cut of a groove of radius $R$ at a height $z$ above the macroscopic meniscus. The liquid forms a sickle-shape lunula of thickness $e$ and area $\Sigma$ (shaded area). $r$ is the lunula free surface radius of curvature.

assumed to be trapped on the groove edges for any value of $e$. For a wetting fluid, this trapping is indeed consistent with the Gibbs' condition [7] for the contact line equilibrium at a solid edge. However, in the case of partial wetting, i.e., for a non zero contact angle $\theta$, there exists a critical thickness $e_{\mathrm{c}}=R\left(1-\frac{1}{\cos \theta}+\operatorname{tg} \theta\right)$ of the lunula below which the anchorage is not possible any more. The lunula thickness $e$ and area $\Sigma$ are related to its radius $r$ by the following expressions :

$$
\begin{gathered}
e=R\left(1-\frac{r}{R}+\sqrt{\left(\frac{r}{R}\right)^{2}-1}\right) \\
\Sigma=R^{2}\left(\frac{\pi}{2}-\left(\frac{r}{R}\right)^{2} \operatorname{Arcsin}\left(\frac{R}{r}\right)+\sqrt{\left(\frac{r}{R}\right)^{2}-1}\right) .
\end{gathered}
$$

Expanding $r$ around $R, r=R(1+\varepsilon)$ with $\varepsilon \ll 1$, the above expressions can be simplified :

$$
\begin{aligned}
& e \simeq R \sqrt{2 \varepsilon} \\
& \Sigma \simeq 2 R e
\end{aligned}
$$

whereas for $\varepsilon \gg 1$ :

$$
\begin{aligned}
& e \simeq R\left\{1-\frac{1}{2}\left(\frac{R}{r}\right)\right\} \\
& \Sigma \simeq R^{2}\left\{\frac{\pi}{2}-\frac{2}{3}\left(\frac{R}{r}\right)\right\} .
\end{aligned}
$$


In the lubrication approximation [8], the mean upward velocity $U$ at height $z$ is given by :

$$
\eta \frac{U}{e^{2}}=-\rho g+\gamma \frac{\partial}{\partial z}\left(\frac{1}{r}\right)
$$

where $\eta$ is the liquid viscosity (numerical factors have been neglected). The first term on the right hand side represents the gravitational pressure gradient while the second is the pressure gradient due to the curvature of the interface. Another relation is provided by the conservation equation :

$$
\frac{\partial \Sigma}{\partial t}+\frac{\partial}{\partial z}(U \Sigma)=0
$$

Combining equations (3) and (4) with (2.a) we get for $\varepsilon \ll 1$

$$
\frac{\partial e}{\partial t}=\frac{\partial}{\partial z}\left(\frac{g \rho}{\eta} e^{3}+\frac{\gamma}{\eta} \frac{e^{4}}{R^{3}} \frac{\partial e}{\partial z}\right) .
$$

The initial conditions for $t=0$ are :

$$
\begin{aligned}
& e(z, t=0)=R \text { for } z=0 \\
& e(z, t=0)=0 \text { for } z>0 .
\end{aligned}
$$

Equation (5) with the above initial conditions describes how the capillary rise takes place in the groove.

Introducing the dimensionless variables

$$
\hat{e}=e / R ; \hat{z}=z / h ; \hat{t}=t / \tau
$$

with

$$
h=\left(\frac{\gamma}{\rho g}\right) \frac{1}{R}=\frac{\kappa^{-2}}{R}
$$

and

$$
\tau=\frac{h^{2}}{\left(\frac{\gamma}{\eta}\right) R}=\frac{h^{2}}{V^{*} R},
$$

(where $V^{*}=\gamma / \eta$ is a characteristic velocity), equation (5) can be rewritten as the following dimensionless equation :

$$
\frac{\partial \hat{e}}{\partial \hat{t}}=\frac{\partial}{\partial \hat{z}}\left(\hat{e}^{3}+\hat{e}^{4} \frac{\partial \hat{e}}{\partial \hat{z}}\right) .
$$

Therefore, $\tau$ is the time scale of the problem.

Before investigating the dynamics of penetration (Sect. 3), we consider in the next section the equilibrium state of the system.

\section{Statics.}

At equilibrium, the upward velocity $U$ of the liquid is equal to zero and the radius of curvature $r$ is related to the vertical coordinate $z$ by (see Eq. (3)) :

$$
\frac{1}{r}=\kappa^{2} z \text {. }
$$


The above relation expresses the balance between the capillary and the gravitational pressures. By setting $r=R$ in equation (9), one finds that the ultimate height of the macroscopic contact line inside the groove is given by $h$ (Eq. (7.a)). It is worth noting that this final height corresponds to half the macroscopic height reached by a wetting fluid in a thin capillary of radius $R$ (Jurin's law) [9]. Combining equations (1.a) and (9) one obtains the equilibrium profile $e=e_{\mathrm{s}}(z)$ of the liquid inside the groove (the subscript $s$ stands for static). In particular, (see Eqs. (2a) and (2b)), for $z \rightarrow h$, the profile is given by :

$$
e_{\mathrm{s}} \simeq R \sqrt{2\left(1-\frac{z}{h}\right)} \quad(z \simeq h)
$$

whereas for $z \rightarrow 0$ :

$$
e_{\mathrm{s}} \simeq R\left(1-\frac{1}{2} \frac{z}{h}\right) \quad(z \simeq 0)
$$

For $z=h$, the slope $\frac{\partial e}{\partial z}$ deduced from (10) is infinite. This is in contradiction with our hypothesis of total wetting. The source of the problem is the following : in equation (3), the vertical curvature of the interface has been neglected. Near $z=h$, this curvature becomes important and does ensure a zero slope. To estimate the size of the region near $z=h$ where the neglected curvature plays an important role, we calculate from (10) the value $z^{*}$ of $z$ where :

$$
\frac{\partial^{2} e_{\mathrm{s}} / \partial^{2} z}{\left[1+\left(\partial e_{\mathrm{s}} / \partial z\right)^{2}\right]^{3 / 2}} \sim R^{-1}
$$

( $R^{-1}$ being the horizontal curvature of the interface at height $h$ ). For typical values of $R$ and $\kappa^{-1}: R \sim 1 \mu \mathrm{m}$ and $\kappa^{-1} \sim 1 \mathrm{~mm}$, one finds :

$$
1-\frac{z^{*}}{h} \leqslant 10^{-4}
$$

Thus, the neglected vertical curvature leads to a departure of the real profile from (10) only in the immediate vicinity of $h$ (see Eq. 12). We now investigate how the equilibrium state calculated in this section is reached by the system.

\section{Dynamics.}

3.1 CAPILlARY RISE IN THE ABSENCE OF GRAVITY. - In the absence of gravity $(g=0)$, equation (5) leads to a nonlinear diffusion equation :

$$
\frac{\partial \hat{e}}{\partial \hat{t}}=\frac{\partial}{\partial \hat{z}}\left(D(\hat{e}) \frac{\partial \hat{e}}{\partial \hat{z}}\right)
$$

with a thickness-dependent diffusion coefficient :

$$
D(\hat{e})=\hat{e}^{4} .
$$

As can be checked, equation (13) admits solutions where $\hat{e}(\hat{z}, \hat{t})$ depends only on the single variable :

$$
y=\hat{z} / \sqrt{\hat{t}}
$$


For this case, equation (13) becomes

$$
\frac{\mathrm{d}^{2} \hat{e}^{5}(y)}{\mathrm{d} y^{2}}+\frac{5}{2} y \frac{\mathrm{d} \hat{e}(y)}{\mathrm{d} y}=0 .
$$

Since the transport of the fluid is very slow at small thickness $(D(\hat{e}) \rightarrow 0$ for $\hat{e} \rightarrow 0)$, the profile $\hat{e}(y)$ is limited to an interval $\left(y=0, y=y_{\mathrm{F}}\right)$ [10]. (The exact value of $y_{\mathrm{F}}$ can be determined by numerical integration of Eq. (16)). This type of profile is called « hypodiffusive » [11]. Assuming a power law behavior for $\hat{e}(y)$ near $y=y_{F}$, one finds, using equation (16) :

$$
\hat{e}(y)=c\left(y_{\mathrm{F}}-y\right)^{1 / 4}
$$

where $c$ is a numerical constant. Going back to the variable $\hat{e}$ and $\hat{t}$ we see that :

- the maximal height $\hat{z}_{\mathrm{F}}$ reached by the fluid follows a diffusion law :

$$
\hat{z}_{\mathrm{F}}(\hat{t})=y_{\mathrm{F}} \sqrt{\hat{t}} \text {. }
$$

- the shape of the front near $\hat{z}_{\mathrm{F}}(\hat{t})$ is given by

$$
\hat{e}(\hat{z} ; \hat{t})=c \hat{t}^{-1 / 8}\left(\hat{z}_{\mathrm{F}}(\hat{t})-\hat{z}\right)^{1 / 4}
$$

Note that the results found in this section may be used as well to describe the flow in an horizontal semi-circular groove.

3.2 CAPILLARY RISE UNDER GRAVITY. - The capillary rise under gravity is governed by the full differential equation (8). In order to solve this equation in the asymptotic regime (i.e. nearby equilibrium), we assume the existence of a crossover point $\Lambda(\hat{t})$ such that (see Fig. 3) :

- for $\hat{z}<\Lambda(\hat{t})$, the profile has already reached its equilibrium shape given by equations (10) and (11).

- for $\hat{z}>\Lambda(\hat{t})$, gravity is negligible and the profile is given by equation (19).

This crossover point $\Lambda(\hat{t})$ can be determined by setting

$$
\hat{e}_{\mathrm{s}}(\hat{z}=\Lambda(\hat{t}))=\hat{e}(\hat{z}=\Lambda(\hat{t}) ; \hat{t})
$$

where the right hand side corresponds to the zero gravity solution (Sect. 3.1). Using equations (10) and (19) one obtains :

$$
\Lambda(\hat{t})=\left(1-\frac{c^{4}}{4 \sqrt{\hat{t}}}\right)+\frac{1}{2} c^{2}\left(\frac{1}{16 \hat{t}}-\frac{1}{\sqrt{\hat{t}}}+1\right)^{1 / 2} .
$$

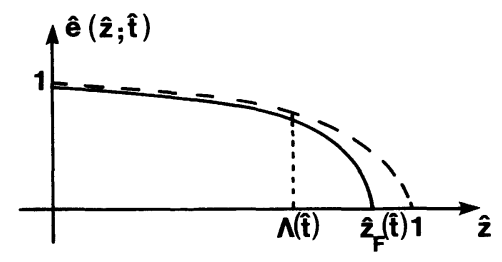

Fig. 3. - Profile of the liquid inside the groove at time $\hat{t}$ (full line). For $\hat{z}<\Lambda(\hat{t})$, the profile has already reached its equilibrium shape (broken line) while for $\hat{z}>\Lambda(\hat{t})$ gravity is negligible and the profile is given by equation (19). 
Since gravity has been assumed to be negligible at the profile tip, the evolution of $\hat{z}_{\mathrm{F}}$ follows a diffusion law (see Sect. 3.1) :

$$
\hat{z}_{\mathrm{F}}(\hat{t})=y_{\mathrm{F}} \sqrt{\hat{t}}
$$

Using equations (21) and (22) we find that

(a) the velocity of $\Lambda(\hat{t})$ is larger than the velocity of $\hat{z}_{\mathrm{F}}(\hat{t})$

(b) the two points $\Lambda(\hat{t})$ and $\hat{z}_{\mathrm{F}}(\hat{t})$ join up at $\hat{z}=1$ for $\hat{t}=\frac{1}{y_{\mathrm{F}}^{2}}$ :

$$
\Lambda\left(\hat{t}=\frac{1}{y_{\mathrm{F}}^{2}}\right)=\hat{z}_{\mathrm{F}}\left(\hat{t}=\frac{1}{y_{\mathrm{F}}^{2}}\right)=1 .
$$

The fluid ascension stops when $\Lambda(\hat{t})$ overtakes $\hat{z}_{\mathrm{F}}(\hat{t})$, i.e. at time $\hat{t}=1 / y_{\mathrm{F}}^{2}$. Due to the neglected curvature in equation (3), our description ceases to be valid when $\hat{e}_{\mathrm{s}}(\hat{z}=\Lambda(\hat{t}))$ becomes smaller than $\hat{e}_{\mathrm{s}}\left(\hat{z}=\hat{z}^{*}\right) \sim 10^{-2}$ (see the discussion in Sect. 3 ).

\section{Conclusions.}

In this paper, an analysis of the capillary rise of a wetting liquid into a vertical semi-circular groove engraved on a wall has been presented. Due to surface tension effects, the fluid rises inside the groove far above the macroscopic meniscus. We have shown in particular that :

- The ultimate height reached by the liquid inside the groove is given by $h=\kappa^{-2} / R$. This height equals half the height reached by a liquid in a close cylindrical capillary of the same radius [9].

- During the fluid ascension, the profile tip follows a diffusion law.

- Typical equilibration time is given by :

$$
\tau=\left(V^{*} R^{3} \kappa^{4}\right)^{-1}
$$

We note that this equilibration time $\tau$ is of the same order of magnitude as the equilibration time in a close cylindrical capillary of radius $R$ [12].

Several extensions of the present work are clearly needed :

- towards grooves of varius geometries;

- towards the incorporation of van der Walls forces.

To our knowledge, quantitative experimental results on the capillary rise in grooves are not available at the present time. Such experiments would require grooves of well controlled geometries. Various optical methods (e.g., ellipsometry) may be used to check the predictions presented in this work.

\section{Acknowledgments.}

I would like to express my gratitude to P.G. de Gennes for invaluable discussions. I have also greatly benefited from conversations with D. Andelman, F. Brochard, R. Bruinsma and J. M. di Meglio. I thank the referees for their attentive reading of the manuscript. 


\section{References}

[1] LuCAS, R., Kolloid Z. 23 (1918) 15.

[2] Washburn, E. W., Phys. Rev. 17 (1921) 273-283.

[3] See, e.g.

Letelier, S. M. F., Leutheusser, H. J. and Rosas, Z. C., J. Colloid Interface Sci. 72 (1979) 465470.

Levine, S., Lowndes, J. and Reed, P., J. Colloid Interface Sci. 77 (1981) 253-263.

MARmur, A., J. Colloid Interface Sci. 122 (1988) 209-219.

[4] Pomeau, Y., J. Colloid Interface Sci. 113 (1985) 5-11.

[5] Lenormand, R. and Zarcone, C., Paper SPE 13264, presented at the 59th Annual Technical Conference of the SPE, Houston, September 16-19 (1984).

[6] Quere, D., Di Meglio, J. M. and Brochard-Wyart, F., Revue Phys. Appl. 23 (1988) 10231030.

[7] GibBs, J. W., Scientific Papers, 1 (1906) 326 ; Dover Reprint, New York (1961).

Oliver, J. F., Huh, C. and Mason, S. G., J. Colloid Interface Sci. 59 (1977) 568-581.

[8] Moffat, K., Fluids Dynamics, Eds. R. Balian and J. L. Peube, (Gordon and Breach, N.Y.) 1973.

[9] BouAsse, H., Capillarité et phénomènes superficiels (Delagrave, Paris) 1924.

[10] DE Gennes, P. G., Phys. Chem. Hydro. 4 (1983) 175-185.

[11] DE GenNes, P. G., Physics of disordered materials, Eds. D. Adler, H. Fritzsche and S. R. Ovshinsky, p. 227-241 (Plenum) 1985.

[12] see, e.g. LeVICH, G., Physicochemical Hydrodynamics (Prentice-Hall) 1962. 